\title{
Alphavirus Encephalomyelitis: Mechanisms and Approaches to Prevention of Neuronal Damage
}

\author{
Diane E. Griffin ${ }^{1}$
}

Published online: 25 April 2016

(C) The American Society for Experimental NeuroTherapeutics, Inc. 2016

\begin{abstract}
Mosquito-borne viruses are important causes of death and long-term neurologic disability due to encephalomyelitis. Studies of mice infected with the alphavirus Sindbis virus have shown that outcome is dependent on the age and genetic background of the mouse and virulence of the infecting virus. Agedependent susceptibility reflects the acquisition by neurons of resistance to virus replication and virus-induced cell death with maturation. In mature mice, the populations of neurons most susceptible to infection are in the hippocampus and anterior horn of the spinal cord. Hippocampal infection leads to long-term memory deficits in mice that survive, while motor neuron infection can lead to paralysis and death. Neuronal death is immune-mediated, rather than a direct consequence of virus infection, and associated with entry and differentiation of pathogenic $\mathrm{T}$ helper 17 cells in the nervous system. To modulate glutamate excitotoxicity, mice were treated with an $N$-methyl-D-aspartate receptor antagonist, $\alpha$-amino-3-hydroxy-5-methyl-4-isoxazole propionic acid receptor antagonists or a glutamine antagonist. The $N$-methyl-D-aspartate receptor antagonist MK-801 protected hippocampal neurons but not motor neurons, and mice still became paralyzed and died. $\alpha$-Amino-3hydroxy-5-methyl-4-isoxazole propionic acid receptor antagonists GYKI-52466 and talampanel protected both hippocampal and motor neurons and prevented paralysis
\end{abstract}

Diane E. Griffin dgriffi6@jhu.edu

1 W. Harry Feinstone Department of Molecular Microbiology and Immunology, Johns Hopkins Bloomberg School of Public Health, Baltimore, MD 21205, USA and death. Glutamine antagonist 6-diazo-5-1-norleucine protected hippocampal neurons and improved memory generation in mice surviving infection with an avirulent virus. Surprisingly, in all cases protection was associated with inhibition of the antiviral immune response, reduced entry of inflammatory cells into the central nervous system, and delayed virus clearance, emphasizing the importance of treatment approaches that include prevention of immunopathologic damage.

Keywords Glutamate excitotoxicity · Immunopathogenesis · Sindbis virus · AMPA receptor antagonist · Glutamine antagonist

\section{Introduction}

Viral encephalomyelitis can be a devastating disease for the infected individual and for society as a whole because those who recover are frequently left with neurological sequelae such as seizures, paralysis, and cognitive deficits [1]. Arthropod-borne (arbo) viruses are important causes of encephalomyelitis with widespread seasonal outbreaks of fever, encephalitis, and arthritis, and pose increasing threats to human populations through continued expansion into new geographic areas $[2,3]$. Alphaviruses are mosquito-borne plus-strandenveloped RNA viruses that cause both encephalomyelitis (Venezuelan, western and eastern equine encephalitis viruses) and arthritis (Sindbis, Ross River, and Chikungunya viruses). The encephalitic alphaviruses are endemic in the Americas, while the rapidly emerging arthritic alphaviruses that can also cause neurologic disease are now found worldwide [2, 4-7]. Currently, 
there are no treatments or vaccines licensed for these infections.

For all arboviruses, severity of disease and outcome after infection varies widely from one person to another. This review will cover our current knowledge of the pathogenesis of fatal encephalomyelitis, the determinants of variable outcome, and potential treatments for severe disease through the study of central nervous system (CNS) infection by the prototype alphavirus, Sindbis virus (SINV), in mice.

\section{Neuronal Damage}

Neurons are the primary target cells of encephalitic alphaviruses and the outcome of infection is determined both by neuronal maturity and infecting virus virulence. Susceptibility to fatal encephalomyelitis diminishes with increasing age [8], and this is not due to changes in the adaptive immune response, but rather to changes in the intrinsic susceptibility of immature and mature neurons to infection [9]. Neuroadapted strains of SINV (e.g., NSV) with improved replication in mature neurons can cause fatal encephalomyelitis in older mice, providing a model system for evaluation of therapeutic interventions. Populations of mature neurons that are particularly susceptible to infection are in the hippocampus and anterior horn of the spinal cord. NSV-infected motor neurons die by a nonapoptotic process [10], and mice develop weakness that progresses to paralysis and death within 7 10 days $[11,12]$.

\section{Role of Neuronal Maturity}

Immature neurons of all types replicate SINV (and other neurotropic arboviruses) to high titers that result in death due to apoptosis, while mature neurons restrict virus replication, are relatively resistant to virusinduced apoptosis, and can become persistently infected (Fig. 1a) [13-15]. Maturation-dependent restriction of virus replication is also observed in cultured primary neurons (e.g., dorsal root ganglia cells) and neuronal cell lines differentiated in vitro (e.g., CSM14.1, AP-7) (Fig. 1b), facilitating mechanistic studies [13, 16-18].

Neuronal maturation in the absence of infection is associated with increased expression of interferon (IFN)- $\beta$, transcription factors IFN regulatory factor (IRF)-3 and IRF-7, and several IFN-stimulated gene (ISG) mRNAs (e.g., 2,5OAS, RNaseL, $\beta 2 \mathrm{~m}$, IFIT1, IFIT3, ISG20) [15]. IRF-7 is a key transcription factor, with multiple splice variants, that regulates and amplifies the IFN response through induction of the IFN- $\alpha$ genes, as well as ISGs [19]. The IRF-7 protein
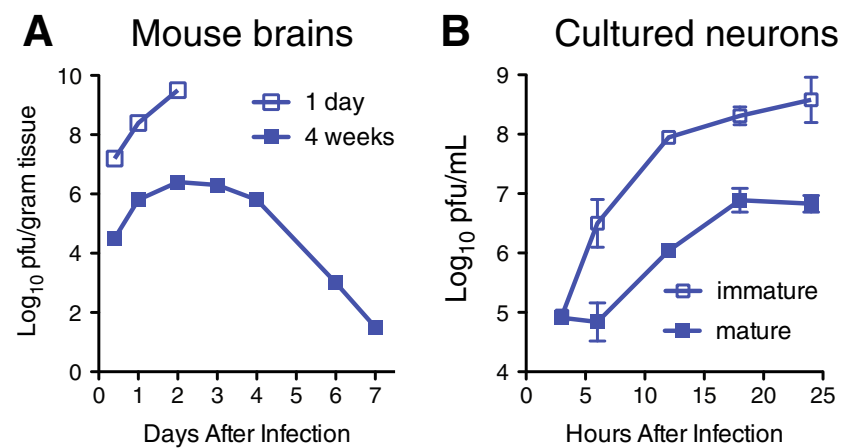

Fig. 1 Neuronal maturation leads to restriction of Sindbis virus replication. a Virus replication in the brains of 1-day and 4-week-old mice after intracerebral inoculation [8]. b Virus replication in immature undifferentiated and mature differentiated AP-7 rat neuronal cells [15]

produced by neurons evolves with maturation from the short dominant negative $\gamma$ isoform to the full-length active $\alpha$ isoform necessary for transcribing antiviral protein genes [15]. With maturation, uninfected neurons produce small amounts of IFN- $\beta$ that results in priming the cell for an antiviral response. Neutralization of IFN increases replication of SINV, suggesting that the low levels of IFN constitutively produced by mature neurons are important for resistance [16]. In response to infection, differentiated, but not undifferentiated, neurons rapidly produce IFN and upregulate ISGs to restrict virus replication. Therefore, neuronal maturation is associated with antiviral priming characterized by increased basal levels of important transcription factors that rapidly activate antiviral signaling in response to infection, and thus reduce virus replication in mature neurons [15].

\section{Role of Virus Strain}

Alphaviruses have a message-sense RNA genome that encodes 4 nonstructural replication proteins (nsP1-4), 3 main structural proteins (capsid and envelope proteins $\mathrm{E} 1$ and $\mathrm{E} 2)$, and 2 small proteins (6 K, TF). NSV is a neuroadapted strain of SINV that is virulent for adult C57BL/6 mice and provides a model for developing an understanding of virus-induced fatal encephalomyelitis in mature animals [20]. NSV has the same neuronal tropism as less virulent strains of SINV but replicates to higher titer and induces more intense inflammation in the brain and spinal cord [11, 21].

Virulence determinants are primarily in the E1 and E2 glycoproteins that regulate virus entry into neurons, alter glycosylation, and change binding to heparan sulfate [22-27]. In addition, recent studies have identified important roles for changes in $\mathrm{nsP} 3, \mathrm{TF}$, and the 5' nontranslated region that influence neuronal replication and alter virulence [28-30]. 


\section{Role of the Immune Response}

Virus clearance from neurons is accomplished through a synergistic process involving T-cell production of IFN- $\gamma$ and B-cell production of antibody to the E2 glycoprotein $[13,31,32]$. Therefore, in response to infection, $\mathrm{T}$ cell-mediated inflammation and B-cell infiltration into the CNS are necessary for virus clearance but need to be regulated to prevent damage to neural tissue [33].

Several observations have led to the conclusion that neuronal damage in mature animals is primarily due to the antiviral immune response rather than virus replication per se, and that fatal alphaviral encephalomyelitis is a T-cell-mediated immunopathologic process. For instance, initiation of virus clearance and the inflammatory response are coincident with the onset of neurological disease [21], and survival is improved in mice deficient in $\alpha \beta \mathrm{T}$ cells, $\beta 2$-microglobulin, transporter associated with antigen processing (TAP), or CD4 but not in mice deficient in production of antibody, CD8, perforin, Fas, TNF- $\alpha$ receptor- 1 , IFN- $\gamma$, IFN- $\gamma$ receptor- 1 , or IL6 [34-36]. Furthermore, mice protected from fatal disease by passive transfer of immune serum after NSV infection clear infectious virus but develop a progressive loss of parenchyma (ex vacuo hydrocephalus) associated with infiltration of $\mathrm{CD}^{+} \mathrm{T}$ cells and macrophages into the hippocampus [35].

IL-10 is an important regulatory cytokine that helps to determine the balance between inflammation and immunoregulation [37, 38]. Deficiency of IL-10 accelerates the onset of fatal NSV-induced paralytic disease with an early increase in the CNS of $\mathrm{CD}^{+} \mathrm{T}$ cells expressing the transcription factor ROR $\gamma \mathrm{t}$ and producing the cytokine IL-17 [T helper (Th) 17 cells] [39]. Th17 cells are multifunctional and can have pathogenic or nonpathogenic characteristics. In response to NSV infection, Th17 cells in the CNS (but not in the draining lymph nodes) had a pathogenic phenotype with production of granulocyte macrophage colony-stimulating factor (GMCSF) and granzyme B. In addition, some Th17 cells in the CNS developed into doubly pathogenic Th1/Th17 cells with additional expression of the transcription factor T-bet and production of IFN- $\gamma$. Although pathogenic Th17 cells are recognized to be effectors in autoimmune disease [40], they were not previously identified as contributors to virus-induced immunopathology [41].

These studies and comparative studies of BALB/c mice that are genetically resistant to fatal NSV-induced encephalomyelitis indicate the importance of IL-10 in regulating the immunopathogenic effects of antiviral $\mathrm{T}$ cells [42]. $\mathrm{CD}^{+} \mathrm{T}$ cells infiltrating the brains of BALB/c mice include fewer Th17 cells and more regulatory $\mathrm{T}$ cells producing $\mathrm{IL}-10$ than similarly infected
C57BL/6 mice [42]. In the absence of IL-10 BALB/c mice become susceptible to fatal infection. The primary sources of regulatory IL-10 during infection are the infiltrating $\mathrm{CD}^{+}$and $\mathrm{CD} 8^{+}$lymphocyte populations, not myeloid cells intrinsic to the CNS [43].

Determination of the role of Th17 cells in NSV-induced immunopathology and identification of the mechanism(s) by which they influence outcome will be important for developing interventions and for identifying host determinants of susceptibility to severe disease. Th17 cells can directly target neurons [44], and under conditions of stress in vitro, neurons express IL17 receptor and treatment with IL-17 can induce neuronal cell death [45]. GM-CSF has also been identified as a potential mediator of neural damage [46-49]. GM-CSF activates microglial cells and recruits myeloid cells into the CNS, but the mechanism by which this leads to disease has not been identified [47, 50, 51]. Furthermore, neutralizing antibody to neither GM-CSF nor IL-17 altered the course of disease compared with control antibody in either IL-10 $0^{-/-}$or wild-type mice [39].

\section{Prevention of Fatal Disease}

Our studies indicate that development of successful treatments for viral encephalomyelitis requires a strategy to decrease immunopathologic damage either as a primary approach or as an adjunct to use of an antiviral drug. Damage to hippocampal and motor neurons suggested that glutamate excitotoxicity might play a role in inducing neuronal death. During infection, excess glutamate may result from extracellular release by damaged neurons or microglial cells [52], production by activated $\mathrm{CD}^{+} \mathrm{T}$ cells entering the CNS [53], or from failure of astrocytes to remove excess glutamate due to a cytokine-induced decrease in expression of glutamate transporter-1 [54, 55]. In vitro treatment of infected primary cortical neurons with $N$-methyl-D-aspartate receptor antagonists MK-801 and D(-)-2-amino-5phosphonopentanoic acid (APV) decreased cell death [56]. Although treatment of NSV-infected mice with MK-801 protected hippocampal neurons, it did not protect motor neurons or prevent paralysis and death of the mice (Fig. 2a) [57]. However, treatment of NSV-infected mice with $\alpha$-amino-3-hydroxy-5-methyl-4-isoxazole propionic acid (AMPA) receptor antagonists GYKI52466 or talampanel protected both hippocampal and motor neurons, improved outcome, and indicated that fatal disease is primarily due to infection of motor neurons rather than hippocampal neurons (Fig. 2a) [57, 58]. Surprisingly, upon examination of the mechanism of protection, it was discovered that AMPA receptor antagonists actually suppressed the antiviral immune response and subsequent entry of inflammatory cells into the 


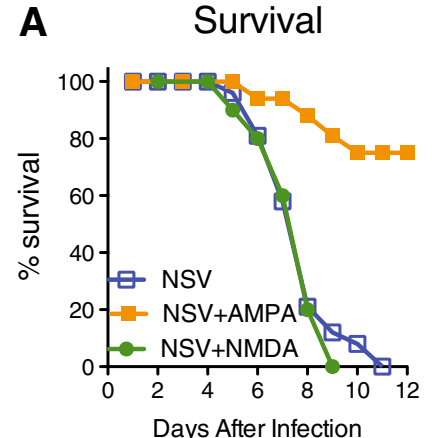

Fig. 2 Effects of treatment of neuroadapted Sindbis virus (NSV)infected mice with glutamate receptor antagonists. a Survival of NSVinfected mice in the absence of treatment and in the presence of treatment for 7 days with the $N$-methyl-D-aspartate (NMDA) receptor antagonist

CNS (Fig. 2b) [57, 58]. Protection occurred despite a resultant delay in virus clearance (Fig. 2c).

Nonfatal SINV infection of hippocampal neurons leads to persistent behavioral abnormalities. At the peak of infectious virus titers in brain (day 5), mice are hyperactive, have decreased anxiety, and memory deficits that persist after clearance of infectious virus and resolution of clinical signs of disease. Mice treated with 6-diazo-5-oxo-1-norleucine, a glutamine antagonist that affects both the immune response by inhibiting lymphocyte proliferation and glutamate excitotoxicity by inhibiting neuronal glutaminase synthesis of glutamate had decreased inflammatory cell infiltration and cell death in the hippocampus [59]. Treatment inhibited development of clinical signs and memory deficits revealed by assessing contextual fear conditioning (Fig. 3), despite the presence of infectious virus and high levels of viral RNA [60].

\section{Future Directions}

For both AMPA receptor and glutamine antagonists, inhibition of the inflammatory response in the CNS prevented fatal disease, despite also slowing virus clearance, further indicating that neuronal damage and fatal

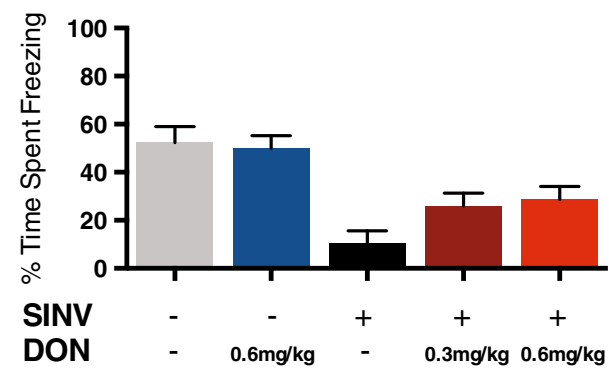

Fig. 3 Effect of treatment with 6-diazo-5-oxo-1-norleucine (DON) on hippocampal-dependent memory in Sindbis virus (SINV)-infected mice. Treatment with DON partially inhibited development of abnormalities in contextual fear conditioning 5 days after intranasal infection with SINV [55]

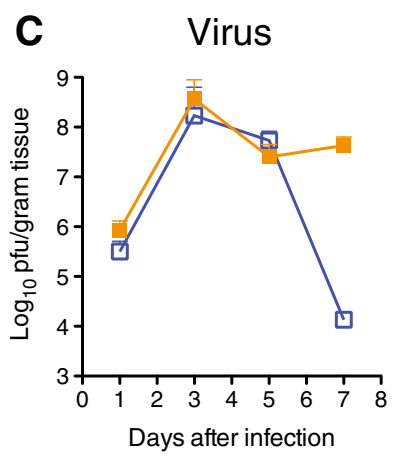

MK-801 or the $\alpha$-amino-3-hydroxy-5-methyl-4-isoxazole propionic acid (AMPA) receptor antagonist GYKI-52466 [52]. Effects of treatment with AMPA receptor antagonist talampanel on T-cell infiltration into the brain $\mathbf{b}$ and on virus replication and clearance from the brain $\mathbf{c}$

disease are due to the immune response to virusinfected neurons rather than virus infection per se. However, when treatment is stopped, the immune response may be initiated along with neurologic disease. Therefore, although there are no approved drugs that inhibit alphavirus replication, ideal treatment would likely combine immune response inhibitors with an antiviral drug.

Acknowledgments Work from the author's laboratory was supported by research grants R01 NS087539 and NS038932 from the National Institutes of Health. The contributions of Victoria K. Baxter, Ivorlyne Greene, Kirsten A. Kulcsar, Sivabalan Manivannan, Jennifer NargiAizenmann, Michelle C. Potter, Kimberly L. W. Schultz, and Barbara S. Slusher to the work described in this review are gratefully acknowledged.

Required Author Forms Disclosure forms provided by the authors are available with the online version of this article.

\section{References}

1. Griffin DE. Emergence and re-emergence of viral diseases of the central nervous system. Prog Neurobiol 2010;91:95-101.

2. Weaver SC, Forrester NL. Chikungunya: Evolutionary history and recent epidemic spread. Antiviral Res 2015;120:32-39.

3. Gubler DJ. The global emergence/resurgence of arboviral diseases as public health problems. Arch Med Res 2002;33:330-342.

4. Chandak NH, Kashyap RS, Kabra D, et al. Neurological complications of Chikungunya virus infection. Neurol India 2009;57:177-180.

5. Renault P, Solet JL, Sissoko D, et al. A major epidemic of chikungunya virus infection on Reunion Island, France, 20052006. Am J Trop Med Hyg 2007;77:727-731.

6. Harley D, Sleigh A, Ritchie S. Ross River virus transmission, infection, and disease: a cross-disciplinary review. Clin Microbiol Rev 2001;14:909-932.

7. Weaver SC, Lecuit M. Chikungunya virus and the global spread of a mosquito-borne disease. N Engl J Med 2015;372:1231-1239.

8. Johnson RT, McFarland HF, Levy SE. Age-dependent resistance to viral encephalitis: studies of infections due to Sindbis virus in mice. J Infect Dis 1972;125:257-262. 
9. Griffin DE. Role of the immune response in age-dependent resistance of mice to encephalitis due to Sindbis virus. J Infect Dis 1976; $133: 456-464$.

10. Havert MB, Schofield B, Griffin DE, Irani DN. Activation of divergent neuronal cell death pathways in different target cell populations during neuroadapted Sindbis virus infection of mice. J Virol 2000;74:5352-5356.

11. Jackson AC, Moench TR, Trapp BD, Griffin DE. Basis of neurovirulence in Sindbis virus encephalomyelitis of mice. Lab Invest 1988;58:503-509.

12. Thach DC, Kimura T, Griffin DE. Differences between C57BL/6 and BALB/cBy mice in mortality and virus replication after intranasal infection with neuroadapted Sindbis virus. J Virol 2000;74: 6156-6161.

13. Levine B, Hardwick JM, Trapp BD, Crawford TO, Bollinger RC, Griffin DE. Antibody-mediated clearance of alphavirus infection from neurons. Science 1991;254:856-860.

14. Levine B, Huang Q, Isaacs JT, Reed JC, Griffin DE, Hardwick JM. Conversion of lytic to persistent alphavirus infection by the bcl-2 cellular oncogene. Nature 1993;361:739-742.

15. Schultz KL, Vernon PS, Griffin DE. Differentiation of neurons restricts Arbovirus replication and increases expression of the alpha isoform of IRF-7. J Virol 2015;89:48-60.

16. Vernon PS, Griffin DE. Characterization of an in vitro model of alphavirus infection of immature and mature neurons. J Virol 2005;79:3438-3447.

17. Ubol S, Levine B, Lee SH, Greenspan NS, Griffin DE. Roles of immunoglobulin valency and the heavy-chain constant domain in antibody-mediated downregulation of Sindbis virus replication in persistently infected neurons. J Virol 1995;69:1990-1993.

18. Burdeinick-Kerr R, Govindarajan D, Griffin DE. Noncytolytic clearance of Sindbis virus infection from neurons by gamma interferon is dependent on Jak/STAT signaling. J Virol 2009;83:3429-3435.

19. Honda K, Yanai H, Negishi H, et al. IRF-7 is the master regulator of type-I interferon-dependent immune responses. Nature 2005;434: 772-777.

20. Griffin DE, Johnson RT. Role of the immune response in recovery from Sindbis virus encephalitis in mice. J Immunol 1977;118: 1070-1075.

21. Jackson AC, Moench TR, Griffin DE, Johnson RT. The pathogenesis of spinal cord involvement in the encephalomyelitis of mice caused by neuroadapted Sindbis virus infection. Lab Invest 1987;56:418-423.

22. Knight RL, Schultz KL, Kent RJ, Venkatesan M, Griffin DE. Role of N-linked glycosylation for Sindbis virus infection and replication in vertebrate and invertebrate systems. J Virol 2009;83:5640-5647.

23. Bear JS, Byrnes AP, Griffin DE. Heparin-binding and patterns of virulence for two recombinant strains of Sindbis virus. Virology 2006;347:183-190.

24. Tucker PC, Griffin DE. Mechanism of altered Sindbis virus neurovirulence associated with a single-amino-acid change in the E2 glycoprotein. J Virol 1991;65:1551-1557.

25. Tucker PC, Lee SH, Bui N, Martinie D, Griffin DE. Amino acid changes in the Sindbis virus E2 glycoprotein that increase neurovirulence improve entry into neuroblastoma cells. J Virol 1997;71:6106-6112.

26. Tucker PC, Strauss EG, Kuhn RJ, Strauss JH, Griffin DE. Viral determinants of age-dependent virulence of Sindbis virus for mice. J Virol 1993;67:4605-4610.

27. Byrnes AP, Griffin DE. Binding of Sindbis virus to cell surface heparan sulfate. J Virol 1998;72:7349-7356.

28. Park E, Griffin DE. The nsP3 macro domain is important for Sindbis virus replication in neurons and neurovirulence in mice. Virology 2009;388:305-314.
29. Park E, Griffin DE. Interaction of Sindbis virus non-structural protein 3 with poly(ADP-ribose) polymerase 1 in neuronal cells. J Gen Virol 2009;90:2073-2080.

30. Snyder JE, Kulcsar KA, Schultz KL, et al. Functional characterization of the alphavirus TF protein. J Virol 2013;87:8511-8523.

31. Binder GK, Griffin DE. Interferon-gamma-mediated site-specific clearance of alphavirus from CNS neurons. Science 2001;293: 303-306.

32. Burdeinick-Kerr R, Wind J, Griffin DE. Synergistic roles of antibody and interferon in noncytolytic clearance of Sindbis virus from different regions of the central nervous system. J Virol 2007;81: $5628-5636$.

33. Couper KN, Blount DG, Riley EM. IL-10: the master regulator of immunity to infection. J Immunol 2008;180:5771-5777.

34. Kimura T, Griffin DE. The role of CD8(+) T cells and major histocompatibility complex class I expression in the central nervous system of mice infected with neurovirulent Sindbis virus. J Virol 2000;74:6117-6125.

35. Kimura T, Griffin DE. Extensive immune-mediated hippocampal damage in mice surviving infection with neuroadapted Sindbis virus. Virology 2003;311:28-39.

36. Rowell JF, Griffin DE. Contribution of T cells to mortality in neurovirulent Sindbis virus encephalomyelitis. J Neuroimmunol 2002;127:106-114.

37. Moore KW, de Waal Malefyt R, Coffman RL, O'Garra A. Interleukin-10 and the interleukin-10 receptor. Annu Rev Immunol 2001;19:683-765.

38. Hedrich CM, Bream JH. Cell type-specific regulation of IL-10 expression in inflammation and disease. Immunol Res 2010;47:185-206.

39. Kulcsar KA, Baxter VK, Greene IP, Griffin DE. Interleukin 10 modulation of pathogenic Th17 cells during fatal alphavirus encephalomyelitis. Proc Natl Acad Sci USA 2014;111:16053-16058.

40. Steinman L. A brief history of $\mathrm{T}(\mathrm{H}) 17$, the first major revision in the $\mathrm{T}(\mathrm{H}) 1 / \mathrm{T}(\mathrm{H}) 2$ hypothesis of $\mathrm{T}$ cell-mediated tissue damage. Nat Med 2007;13:139-145.

41. Steinman L. Going viral and the fatal vulnerability of neurons from immunity, not from infection. Proc Natl Acad Sci USA 2014;111: 16982-16983.

42. Kulcsar KA, Baxter VK, Abraham R, Nelson A, Griffin DE. Distinct immune responses in resistant and susceptible strains of mice during neurovirulent alphavirus encephalomyelitis. J Virol 2015;89:8280-8291.

43. Kulcsar KA, Griffin DE. T cell-derived interleukin-10 is an important regulator of the Th17 response during lethal alphavirus encepalomyelitis. J Neuroimmunol 2016. doi:10.1016/jneuroim. 2016.04.010.

44. Siffrin $\mathrm{V}$, Radbruch $\mathrm{H}$, Glumm $\mathrm{R}$, et al. In vivo imaging of partially reversible Th17 cell-induced neuronal dysfunction in the course of encephalomyelitis. Immunity 2010;33:424-436.

45. Wang DD, Zhao YF, Wang GY, et al. IL-17 potentiates neuronal injury induced by oxygen-glucose deprivation and affects neuronal IL-17 receptor expression. J Neuroimmunol 2009;212:17-25.

46. Lee Y, Awasthi A, Yosef N, et al. Induction and molecular signature of pathogenic TH17 cells. Nat Immunol 2012;13:991-999.

47. Codarri L, Gyulveszi G, Tosevski V, et al. RORgammat drives production of the cytokine GM-CSF in helper T cells, which is essential for the effector phase of autoimmune neuroinflammation. Nat Immunol 2011;12:560-567.

48. Kebir H, Kreymborg K, Ifergan I, et al. Human TH17 lymphocytes promote blood-brain barrier disruption and central nervous system inflammation. Nat Med 2007;13:1173-1175.

49. McQualter JL, Darwiche R, Ewing C, et al. Granulocyte macrophage colony-stimulating factor: a new putative therapeutic target in multiple sclerosis. J Exp Med 2001;194:873-882. 
50. El-Behi M, Ciric B, Dai H, et al. The encephalitogenicity of T(H)17 cells is dependent on IL-1- and IL-23-induced production of the cytokine GM-CSF. Nat Immunol 2011;12:568-575.

51. Ponomarev ED, Shriver LP, Maresz K, Pedras-Vasconcelos J, Verthelyi D, Dittel BN. GM-CSF production by autoreactive T cells is required for the activation of microglial cells and the onset of experimental autoimmune encephalomyelitis. J Immunol 2007;178:39-48.

52. Chen CJ, Ou YC, Chang CY, et al. Glutamate released by Japanese encephalitis virus-infected microglia involves TNFalpha signaling and contributes to neuronal death. Glia 2012;60:487-501.

53. Melzer N, Hicking G, Bittner S, et al. Excitotoxic neuronal cell death during an oligodendrocyte-directed CD8+ T cell attack in the CNS gray matter. J Neuroinflam 2013;10:121.

54. Carmen J, Rothstein JD, Kerr DA. Tumor necrosis factoralpha modulates glutamate transport in the CNS and is a critical determinant of outcome from viral encephalomyelitis. Brain Res 2009;1263:143-154.
55. Darman J, Backovic S, Dike S, et al. Viral-induced spinal motor neuron death is non-cell-autonomous and involves glutamate excitotoxicity. J Neurosci 2004;24:7566-7675.

56. Nargi-Aizenman JL, Griffin DE. Sindbis virus-induced neuronal death is both necrotic and apoptotic and is ameliorated by N-methyl-Daspartate receptor antagonists. J Virol 2001;75:7114-7121.

57. Nargi-Aizenman JL, Havert MB, Zhang M, Irani DN, Rothstein JD, Griffin DE. Glutamate receptor antagonists protect from virusinduced neural degeneration. Ann Neurol 2004;55:541-549.

58. Greene IP, Lee EY, Prow N, Ngwang B, Griffin DE. Protection from fatal viral encephalomyelitis: AMPA receptor antagonists have a direct effect on the inflammatory response to infection. Proc Natl Acad Sci USA 2008;105:3575-3580.

59. Thangavelu K, Chong QY, Low BC, Sivaraman J. Structural basis for the active site inhibition mechanism of human kidney-type glutaminase (KGA). Sci Rep 2014;4:3827.

60. Potter MC, Baxter VK, Mathey RW, et al. Neurological sequelae induced by alphavirus infection of the CNS are attenuated by treatment with the glutamine antagonist 6-diazo-5-oxo-1-norleucine. J Neurovirol 2015;21:159-173. 